\title{
Applying the termination of resuscitation rules to out-of-hospital cardiac arrests of both cardiac and non-cardiac etiologies: a prospective cohort study
}

Masahiro Kashiura ${ }^{1 *} \mathbb{D}$, Yuichi Hamabe ${ }^{1}$, Akiko Akashi ${ }^{1}$, Atsushi Sakurai ${ }^{2}$, Yoshio Tahara ${ }^{3}$, Naohiro Yonemoto ${ }^{4}$, Ken Nagao ${ }^{5}$, Arino Yaguchi ${ }^{6}$, Naoto Morimura ${ }^{7}$ and on behalf of the SOS-KANTO 2012 Study Group

\begin{abstract}
Background: The 2015 American Heart Association Guidelines for Cardiopulmonary Resuscitation recommend Basic Life Support (BLS) and Advanced Life Support (ALS) rules for termination of resuscitation (TOR). However, it is unclear whether the TOR rules are valid for out-of-hospital cardiac arrests (OHCAs) of both cardiac and non-cardiac etiologies. In this study, we validated the TOR rules for OHCA resulting from both etiologies.

Methods: This was a prospective multicenter observational study of OHCA patients transported to 67 emergency hospitals between January 2012 and March 2013 in the Kanto region of Japan. We calculated the specificity and positive predictive value (PPV) for neurologically unfavorable outcomes at one month in patients with OHCA of cardiac and non-cardiac etiologies.

Results: Of 11,505 eligible cases, 6,138 and 5,367 cases were of cardiac and non-cardiac etiology, respectively. BLS was performed on 2,818 and 2,606 patients with OHCA of cardiac and non-cardiac etiology, respectively. ALS was performed on 3,320 and 2,761 patients with OHCA of cardiac and non-cardiac etiology, respectively. The diagnostic accuracy of the TOR rules for predicting unfavorable outcomes in patients with OHCA of cardiac etiology who received BLS included a specificity of 0.985 (95\% confidence interval [CI]: 0.956-0.997) and a PPV of 0.999 (95\% Cl: 0.996-1.000). In patients with OHCA from cardiac etiologies who received ALS, the TOR rules had a specificity of 0.963 (95 \% Cl: 0.896-0.992) and a PPV of 0.997 (95 \% Cl: 0.991-0.999). In patients with OHCA from non-cardiac etiologies who received BLS, the specificity was 0.915 (95 \% Cl: 0.796-0.976) and PPV was 0.998 (95\% Cl: 0.995-0.999). For patients with OHCA from non-cardiac etiologies who received ALS, the specificity was 0.833 (95\% Cl: $0.586-0.964$ ) and PPV was 0.996 (95 \% Cl: 0.988-0.999).

Conclusions: Both TOR rules have high specificity and PPV in patients with OHCA from cardiac etiologies. For patients with OHCA from non-cardiac etiologies, the rules had a high PPV, but relatively low specificity. Therefore, TOR rules are useful in patients with OHCA from cardiac etiologies, but should be applied with caution to patients with OHCA from non-cardiac etiologies.
\end{abstract}

Keywords: Cardiopulmonary resuscitation, Decision support techniques, Emergency medical services, Out-of-hospital cardiac arrest

\footnotetext{
* Correspondence: kashiura@me.com

${ }^{1}$ Tertiary Emergency Medical Center, Tokyo Metropolitan Bokutoh Hospital,

4-23-15 Kotobashi, Sumida-ku 130-8575Tokyo, Japan

Full list of author information is available at the end of the article
}

\section{Ciomed Central}

(C) 2016 Kashiura et al. Open Access This article is distributed under the terms of the Creative Commons Attribution 4.0 International License (http://creativecommons.org/licenses/by/4.0/), which permits unrestricted use, distribution, and reproduction in any medium, provided you give appropriate credit to the original author(s) and the source, provide a link to the Creative Commons license, and indicate if changes were made. The Creative Commons Public Domain Dedication waiver (http://creativecommons.org/publicdomain/zero/1.0/) applies to the data made available in this article, unless otherwise stated. 


\section{Background}

Out-of-hospital cardiac arrest (OHCA) is a major public health problem worldwide. Approximately 330,000 individuals in the United States and 275,000 individuals in Europe experience OHCAs each year [1, 2]. The survival rate has steadily improved in OHCA cases from cardiac etiologies, whereas the survival rate for OHCA from all etiologies is about $10 \%$, similar to the rate three decades ago [1, 3, 4]. Most patients who survive an OHCA are resuscitated in the prehospital setting and are subsequently transported to emergency hospitals. Transporting all OHCA patients to emergency hospitals results in unnecessary consumption of valuable resources and exposes paramedics and the public to the risks of high-speed transportation [5].

Several studies have been conducted to prospectively determine and test the unequivocal termination of resuscitation (TOR) rules. The 2015 American Heart Association (AHA) Guidelines Update for Cardiopulmonary Resuscitation and Emergency Cardiovascular Care recommends that emergency medical service (EMS) personnel in prehospital settings should follow TOR rules in the protocols for basic life support (BLS) and advanced life support (ALS) [6]. The BLS TOR rule has three criteria, and all three of the following criteria must be present before terminating BLS resuscitative attempts for adult patients with OHCA: arrest was not witnessed by EMS personnel; no return of spontaneous circulation (ROSC) in the field; and no shock was delivered [7]. The ALS TOR rule recommends considering terminating resuscitation efforts when all of the following four criteria are met in the field: arrest was not witnessed; bystander cardiopulmonary resuscitation (CPR) was not provided; no ROSC after ALS care in the field; and no shock was delivered [8].

In a prospective study, the BLS TOR rule was $100 \%$ predictive of death [9]. In several validation studies, both TOR rules were reported to have a high specificity and positive predictive value (PPV) for death or poor neurological outcomes $[7,10,11]$. The implementation of the TOR rules significantly reduces the rate of transport of futile OHCA. However, the European Resuscitation Council (ERC) guideline challenged the TOR rules and argued that applying the TOR rules led to an unexpected survival of 3.4-9 \% of OHCA patients without sustained ROSC in the prehospital setting $[12,13]$. Moreover, these validation studies were performed in patients with OHCA of presumed cardiac etiology and excluded patients with OHCA from noncardiac etiologies, such as suffocation, pulmonary embolism, incidental hypothermia, and vascular disease [7, 9-11]. Although the TOR rules have been validated in patients with OHCA from all etiologies in small population studies, there have been no studies specifically focused on OHCA of noncardiac etiology $[14,15]$.
As a result, it remains unclear whether the TOR rules are useful in patients with OHCA both of cardiac and noncardiac etiologies.

In this study, we assessed the validity of the BLS and ALS TOR rules for patients suffering from OHCA of cardiac or noncardiac etiology using data from the Survey of Survivors after Cardiac Arrest, which was collected in the Kanto Area of Japan in 2012 (SOS-KANTO 2012).

\section{Methods}

\section{Setting and design}

SOS-KANTO 2012 is a prospective, multicenter (including 67 emergency hospitals) observational study conducted between January 2012 and March 2013 in the Kanto region of Japan. The design and data collection methods used in SOS-KANTO 2012 have been reported in detail in prior studies $[16,17]$. The Kanto region is made up of primarily urban areas, including Tokyo. The institutional ethics committees of each participating institution approved the study with a waiver for informed consent in order to protect participant anonymity.

\section{The EMS system in Japan}

The EMS system in Japan has been explained in several previous studies $[11,18]$. The EMS system is supervised by the Fire and Disaster Management Agency of Japan and is operated by each municipal government. All EMS personnel are certified to perform CPR according to the Japanese resuscitation guidelines produced by the Japan Resuscitation Council. Generally, the ambulance crew consists of three EMS personnel, including at least one emergency lifesaving technician (ELST). ELSTs may perform several resuscitation methods under the supervision of online medical control, including the operation of a semiautomated external defibrillator, insertion of a supraglottic airway device, and insertion of a peripheral intravenous line. Specially trained ELSTs have been able to perform endotracheal intubation since 2004 and to administer adrenaline intravenously under the supervision of online medical control since 2006. EMS personnel in Japan are not legally permitted to terminate resuscitation in the field, and all OHCA patients are transported to hospitals, except in cases where death is certain $[18,19]$.

\section{Participants}

All OHCA patients transported by EMS personnel to participating institutions during the study were eligible for inclusion in SOS-KANTO 2012. Of these patients, the present study included only adults (18 years or older) suffering from nontraumatic OHCA. In addition, the following cases were excluded: cases with missing data regarding inclusion criteria or main outcomes (e.g., age, etiology of cardiac arrest, outcomes 1 month after 
cardiac arrest); cases with initial resuscitation performed inside the hospital and/or missing data for onset location; cases in which it was unknown whether BLS or ALS was performed by EMS personnel; and cases with missing data regarding BLS/ALS TOR criteria. Eligible participants were divided into two groups: those with OHCA from cardiac etiologies, and those with OHCA from noncardiac etiologies. Cases involving BLS and ALS were evaluated according to the BLS and ALS TOR rules, respectively.

\section{Data collection and definition}

The EMS personnel collected prehospital data based on the Utstein-style template [20]. The absence of prehospital ROSC, one component of the TOR rule, was defined as no ROSC despite resuscitation effort of the EMS personnel until hospital arrival. Physicians collected data regarding in-hospital treatments and outcomes, and determined the etiology of OHCA, including cardiac (presumed cardiac origin) and noncardiac (e.g., asphyxia, trauma, aortic disease, drawing, cerebrovascular disease, and drug overdose). If more than one etiology was possible (e.g., ventricular fibrillation arrest leading to a fall from height), the most likely primary cause was recorded at the discretion of physicians.

\section{Outcome measurements}

The primary outcome was survival with favorable neurological outcomes 1 month after cardiac arrest, defined as a Glasgow-Pittsburgh cerebral-performance category of 1 (good performance) or 2 (moderate disability) on a five-category scale $[21,22]$. The other categories of 3 (severe disability), 4 (vegetative state), and 5 (death) were defined as unfavorable neurological outcomes. The secondary outcome was mortality 1 month after cardiac arrest.

\section{Statistical analysis}

Descriptive statistics were calculated for all variables of interest. Continuous variables were reported as means and standard deviations, whereas categorical variables were summarized using counts and percentages. The BLS and ALS TOR rules were evaluated as diagnostic tests. The test characteristics, including sensitivity, specificity, false-positive rate (FPR), PPV and negative predictive value, were reported using $95 \%$ confidence intervals (CIs). Lower sensitivity meant that more patients, who eventually died, were predicted to have good outcomes. AHA and ERC guidelines recommend the FPR to be close to $0 \%$ and classify the reporting imprecision of a diagnostic test for prognostication as serious when the upper limit of the $95 \%$ CI is $>10 \%[23,24]$. Statistical analysis was performed using IBM SPSS for Mac Version 22.0 (IBM Corp., Armonk, NY, USA).

\section{Results}

The study flow chart is shown in Fig. 1. During the survey period, a total of 16,452 patients with OHCA were documented. Of these, the following cases were excluded: 288 patients aged <18 years, 1075 patients with traumatic OHCA, 494 patients with missing etiology of arrest data, 239 cases with missing outcome data, 260 patients who initially received CPR in the hospital, and 230 cases with missing data for onset location. Therefore, 13,866 cases of nontraumatic adult OHCA were eligible for inclusion. However, an additional 724 cases were excluded because it was unknown whether BLS or ALS had been performed, and 1637 cases were excluded due to missing data regarding the TOR criteria (12 cases, unknown witnessed events; 106 cases, unknown shock delivery; and 1519 cases, unknown prehospital ROSC). As a result, 11,505 patients were enrolled in the present study. These patients were divided into two groups, with 6138 cases of OHCA from presumed cardiac etiologies and 5367 cases of OHCA from noncardiac etiologies. In total, 5424 patients received BLS (2818 cardiac cases and 2606 noncardiac cases) and 6081 patients received ALS (3320 cardiac cases and 2761 noncardiac cases).

The patient characteristics are presented in Table 1. The mean patient age was 72.4 years, and 6884 patients were men (59.8\%). Cardiac arrest occurred at home in 8395 cases $(73.0 \%)$. The mean time from call to ambulance arrival on the scene was 8.0 minutes, and the mean time from call to hospital arrival was 35.3 minutes. In 845 cases $(7.3 \%)$, the initial observed rhythm upon arrival was ventricular fibrillation or ventricular tachycardia. Advanced airways were placed in 5368 patients (46.7\%), and adrenaline was administered to 2287 patients (19.9\%). In total, BLS was performed on 5424 patients (47.1\%) and ALS was performed on 6081 patients $(52.9 \%)$.

Regarding the TOR rules criteria, cardiac arrests were witnessed by bystanders and EMS personnel in $5473(47.6 \%)$ cases and 929 (8.1 \%) cases, respectively. Prehospital defibrillation was delivered in 1565 cases $(13.6 \%)$, bystander-initiated CPR was performed in 4231 cases (36.8\%), and ROSC was achieved in the prehospital setting in 1070 cases (9.3\%). In summary, 4193 and 1701 patients met all BLS and ALS TOR criteria, respectively.

Characteristics regarding in-hospital treatment and outcome data are presented in Table 2. Shocks were delivered in the emergency room in 1144 cases (9.9\%), and adrenaline was administered in 9086 cases (79.0\%). Of the 2321 patients $(20.2 \%)$ who survived until hospital admission, coronary angiography and therapeutic hypothermia was performed in 520 cases $(22.4 \%$ ) and 606 cases (26.1\%), respectively. One month after admission, 11,161 cases 


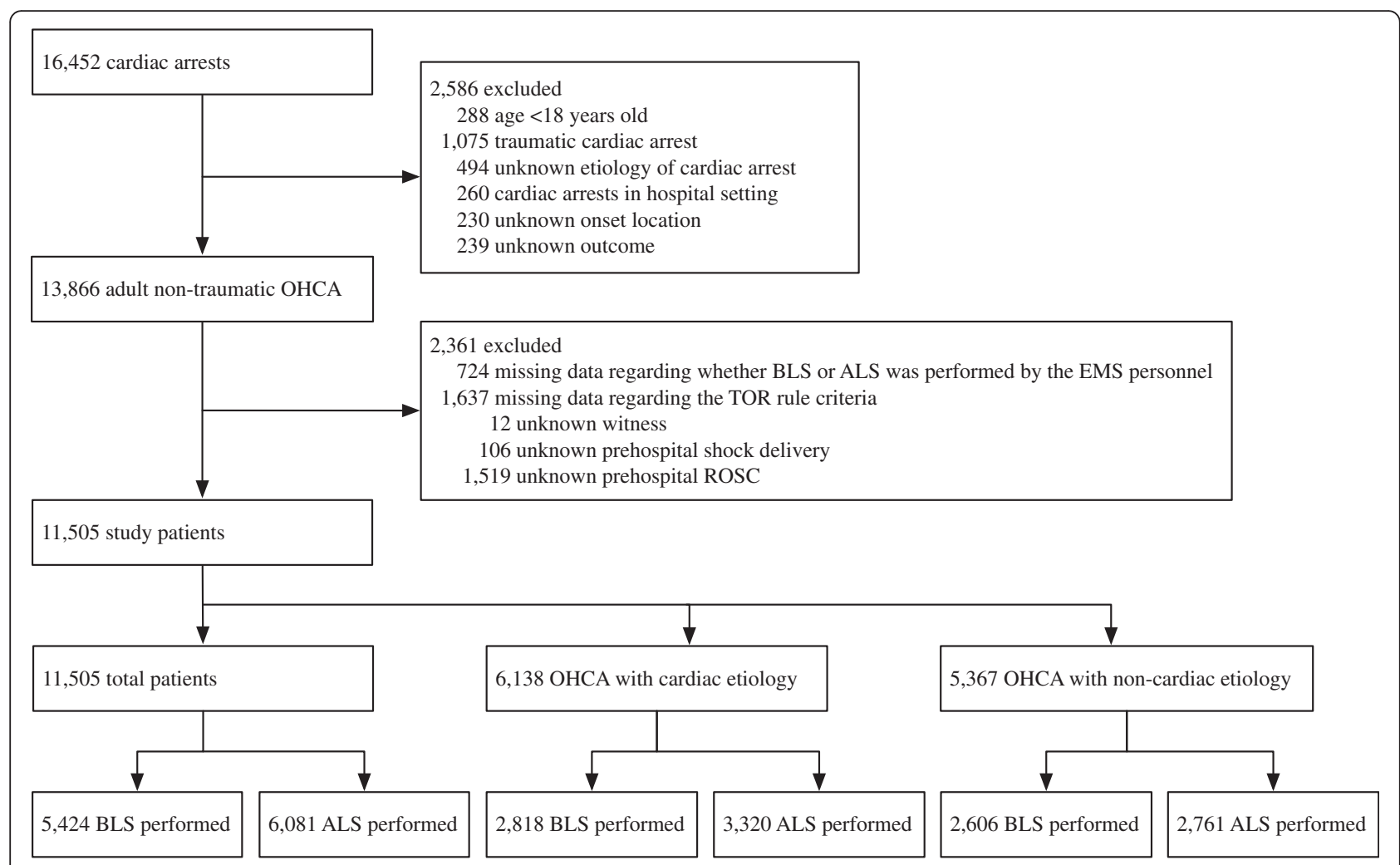

Fig. 1 Flow diagram of the study population. The population for this study was obtained from data in the Survey of Survivors after Cardiac Arrest, which were collected in the Kanto Area of Japan from January 2012 to March 2013. ALS advanced life support, BLS basic life support, EMS emergency medical service, OHCA out-of-hospital cardiac arrest, ROSC return of spontaneous circulation, TOR termination of resuscitation

(97.0\%) had unfavorable neurological outcomes, and 10,933 patients $(95.0 \%)$ had died.

\section{Diagnostic accuracy of the TOR rules for all patients}

The characteristics of the diagnostic tests for the TOR rules for 1-month neurological outcomes and mortality are presented in Tables 3 and 4, respectively. For all cases (OHCA from both cardiac and noncardiac etiologies), the BLS TOR rule had a specificity of 0.971 (95\% CI: $0.942-0.988$ ), a FPR of 2.9 \% (95 \% CI: 1.2-5.8 \%), and a PPV of 0.998 (95 \% CI: 0.997-0.999) for predicting neurologically unfavorable outcomes at 1 month (Table 3 ). In this same group, the BLS TOR rules had a specificity of 0.918 (95 \% CI: 0.884-0.945), a FPR of $8.2 \%$ (95\% CI: 5.5-11.6\%), and a PPV of 0.993 (95 \% CI: 0.990-0.996) for 1-month mortality (Table 4). The ALS TOR rule in patients with OHCA from both cardiac and noncardiac etiologies had a specificity of 0.939 (95 \% CI: 0.8730.977), a FPR of $6.1 \%$ (95 \% CI: $2.3-12.7 \%$ ), and a PPV of 0.996 (95 \% CI: 0.992-0.999) for predicting neurologically unfavorable outcomes at 1 month (Table 3). The ALS TOR rule had a specificity of 0.913 (95 \% CI: 0.8690.946), a FPR of $8.7 \%$ (95 \% CI: 5.4-13.1\%), and a PPV of 0.988 (95 \% CI: 0.982-0.993) for 1-month mortality (Table 4).

\section{Diagnostic accuracy of the TOR rules for patients with OHCA of cardiac etiology}

In patients with OHCA from cardiac etiologies, the BLS TOR rule had a specificity of 0.985 (95 \% CI: 0.9560.997), a FPR of $1.5 \%$ (95 \% CI: 0.3-4.4\%), and a PPV of 0.999 (95 \% CI: 0.996-1.000) for predicting neurologically unfavorable outcomes at 1 month (Table 3 ). In this same group, the BLS TOR rules had a specificity of 0.938 (95 \% CI: 0.901-0.964), a FPR of $6.2 \%$ (95 \% CI: 3.6-9.9\%), and a PPV of 0.992 (95 \% CI: 0.987-0.996) for 1-month mortality (Table 4). The ALS TOR rule for patients with OHCA from cardiac etiologies had a specificity of 0.963 (95\% CI: 0.896-0.992), a FPR of $3.7 \%$ (95 \% CI: $0.8-10.4 \%$ ), and a PPV of 0.997 (95\% CI: 0.991-0.999) for predicting neurologically unfavorable outcomes at 1 month (Table 3). For 1-month mortality, the ALS TOR rule had a specificity of 0.941 (95\% CI: 0.891-0.973), a FPR of $5.9 \%$ (95 \% CI: 2.7-10.9\%), and a PPV of 0.991 (95\% CI: 0.982-0.996) (Table 4).

\section{Diagnostic accuracy of the TOR rules for patients with OHCA of noncardiac etiology}

In patients with OHCA from noncardiac etiologies, the BLS TOR rule had a specificity of 0.915 (95\% CI: 0.796-0.976), a FPR of $8.5 \%$ (95 \% CI: 2.4-20.4\%), and 
Table 1 Clinical and prehospital characteristics of out-of-hospital cardiac arrest patients by group

\begin{tabular}{|c|c|c|c|}
\hline Characteristic & Total $(n=11,505)$ & Cardiac etiology $(n=6138)$ & Noncardiac etiology $(n=5367)$ \\
\hline Age (years) & $72.4 \pm 15.8$ & $73.4 \pm 14.5$ & $71.2 \pm 17.1$ \\
\hline Male & $6884(59.8)$ & $3827(62.3)$ & $3057(57.0)$ \\
\hline \multicolumn{4}{|l|}{ Location of arrest } \\
\hline Home & $8395(73.0)$ & $4365(71.1)$ & $4030(75.1)$ \\
\hline Public space (indoor) & $352(3.1)$ & $217(3.5)$ & $135(2.5)$ \\
\hline Public space (outdoor) & $622(5.4)$ & $435(7.1)$ & $187(3.5)$ \\
\hline Other & $2136(18.6)$ & $1121(18.3)$ & $1015(18.9)$ \\
\hline \multicolumn{4}{|l|}{ EMS care interval (minutes) } \\
\hline Call to arrival of ambulance at scene & $8.0 \pm 3.6$ & $8.0 \pm 3.7$ & $7.9 \pm 3.6$ \\
\hline Call to hospital arrival & $35.3 \pm 3.6$ & $35.4 \pm 11.0$ & $35.3 \pm 12.0$ \\
\hline \multicolumn{4}{|l|}{ Initial rhythm } \\
\hline Ventricular fibrillation/ventricular tachycardia & $845(7.3)$ & $752(12.3)$ & $93(1.7)$ \\
\hline Pulseless electrical activity & $2369(20.6)$ & $1111(18.1)$ & $1258(23.4)$ \\
\hline Asystole & $7550(65.6)$ & $3909(63.7)$ & $3641(67.8)$ \\
\hline Others or unknown & $741(6.4)$ & $366(6.0)$ & $375(7.0)$ \\
\hline Advanced airway procedure & $5368(46.7)$ & $2941(47.9)$ & $2427(45.2)$ \\
\hline Prehospital adrenaline administration & $2287(19.9)$ & $1220(19.9)$ & $1067(19.9)$ \\
\hline BLS performed by EMS & $5424(47.1)$ & $2818(46.9)$ & 2606 (48.6) \\
\hline Met BLS TOR criteria & 4193 & 2060 & 2133 \\
\hline ALS performed by EMS & $6081(52.9)$ & $3320(54.1)$ & $2761(51.4)$ \\
\hline Met ALS TOR criteria & 1701 & 956 & 745 \\
\hline \multicolumn{4}{|l|}{ Arrest witnessed by } \\
\hline Bystander & $5473(47.6)$ & $2881(46.9)$ & $2592(48.3)$ \\
\hline EMS/first responder & $929(8.1)$ & $480(7.8)$ & $449(8.4)$ \\
\hline Prehospital defibrillation (layperson and EMS) & 1565 (13.6) & $1249(20.3)$ & $316(5.9)$ \\
\hline Bystander-initiated CPR & $4231(36.8)$ & $2226(36.3)$ & 2005 (37.4) \\
\hline Prehospital ROSC & 1070 (9.3) & $570(9.3)$ & $500(9.3)$ \\
\hline
\end{tabular}

Data presented as means \pm standard deviations for continuous variables and numbers (percentage) for categorical values

$A L S$ advanced life support, BLS basic life support, CPR cardiopulmonary resuscitation, EMS emergency medical service, $R O S C$ return of spontaneous circulation, TOR termination of resuscitation

Table 2 In-hospital management and 1-month outcomes for out-of-hospital cardiac arrest patients by group

\begin{tabular}{llll}
\hline Characteristic & Total $(n=11,505)$ & Cardiac etiology $(n=6138)$ & Noncardiac etiology $(n=5367)$ \\
\hline Defibrillation in ER & $1144(9.9)$ & $831(13.5)$ & $313(5.8)$ \\
Adrenaline administration in ER & $9086(79.0)$ & $4819(78.5)$ & $4267(79.5)$ \\
Survival until hospital admission & $2321(20.2)$ & $1112(18.1)$ & $1209(22.5)$ \\
Coronary angiography $^{a}$ & $520(22.4)$ & $483(43.4)$ & $37(3.1)$ \\
Therapeutic hypothermia & & $437(39.3)$ & $169(14.0)$ \\
Clinical outcomes 1 month after admission & $606(26.1)$ & & $65(1.2)$ \\
$\quad$ & & $279(4.5)$ & $5302(98.8)$ \\
$\quad$ Neurologically favorable outcome (CPC 1 or 2) & $344(3.0)$ & $5859(95.5)$ & $162(3.0)$ \\
$\quad$ Neurologically unfavorable outcome (CPC 3-5) & $11,161(97.0)$ & $410(6.7)$ & $5205(97.0)$ \\
$\quad$ Survival & $572(5.0)$ & $5728(93.3)$ &
\end{tabular}

Data presented as numbers (percentage) for categorical values

aproportion calculated using the numbers of patients who survived until hospital admission as the denominator

$C P C$ cerebral performance category, ER emergency room 
Table 3 Diagnostic accuracy of termination of resuscitation rules for 1-month neurological outcomes

\begin{tabular}{|c|c|c|c|c|c|c|c|}
\hline $\begin{array}{l}\text { Etiology of } \\
\text { cardiac arrest }\end{array}$ & $\begin{array}{l}\text { Neurologically } \\
\text { unfavorable } \\
\text { outcome }\end{array}$ & $\begin{array}{l}\text { Neurologically } \\
\text { favorable } \\
\text { outcome }\end{array}$ & Sensitivity (95 \% CI) & Specificity (95 \% Cl) & FPR $(95 \%$ Cl) & PPV (95 \% Cl) & NPV $(95 \% \mathrm{Cl})$ \\
\hline \multicolumn{8}{|l|}{ BLS TOR rule } \\
\hline \multicolumn{8}{|l|}{ All types } \\
\hline Met criteria & 4186 & 7 & 0.808 & 0.971 & $2.9 \%$ & 0.998 & 0.193 \\
\hline Did not meet criteria & 993 & 238 & $(0.797-0.819)$ & $(0.942-0.988)$ & $(1.2-5.8 \%)$ & $(0.997-0.999)$ & $(0.172-0.217)$ \\
\hline \multicolumn{8}{|l|}{ Cardiac etiology } \\
\hline Met criteria & 2057 & 3 & 0.785 & 0.985 & $1.5 \%$ & 0.999 & 0.257 \\
\hline Did not meet criteria & 563 & 195 & $(0.769-0.801)$ & $(0.956-0.997)$ & $(0.3-4.4 \%)$ & $(0.996-1.000)$ & $(0.226-0.290)$ \\
\hline \multicolumn{8}{|l|}{ Noncardiac etiology } \\
\hline Met criteria & 2129 & 4 & 0.832 & 0.915 & $8.5 \%$ & 0.998 & 0.091 \\
\hline Did not meet criteria & 430 & 43 & $(0.817-0.846)$ & $(0.796-0.976)$ & $(2.4-20.4 \%)$ & (0.995-0.999) & $(0.067-0.121)$ \\
\hline \multicolumn{8}{|l|}{ ALS TOR rule } \\
\hline \multicolumn{8}{|l|}{ All types } \\
\hline Met criteria & 1695 & 6 & 0.353 & 0.939 & $6.1 \%$ & 0.996 & 0.021 \\
\hline Did not meet criteria & 4287 & 93 & $(0.341-0.365)$ & $(0.873-0.977)$ & $(2.3-12.7 \%)$ & $(0.992-0.999)$ & $(0.017-0.026)$ \\
\hline \multicolumn{8}{|l|}{ Cardiac etiology } \\
\hline Met criteria & 953 & 3 & 0.294 & 0.963 & $3.7 \%$ & 0.997 & 0.033 \\
\hline Did not meet criteria & 2286 & 78 & $(0.279-0.310)$ & $(0.896-0.992)$ & $(0.8-10.4 \%)$ & (0.991-0.999) & $(0.026-0.041)$ \\
\hline \multicolumn{8}{|l|}{ Noncardiac etiology } \\
\hline Met criteria & 742 & 3 & 0.271 & 0.833 & $16.7 \%$ & 0.996 & 0.007 \\
\hline Did not meet criteria & 2001 & 15 & $(0.254-0.288)$ & $(0.586-0.964)$ & (3.6-41.4 \%) & (0.988-0.999) & $(0.004-0.012)$ \\
\hline
\end{tabular}

ALS advanced life support, BLS basic life support, CI confidence interval, FPR false-positive rate, NPV negative predictive value, PPV positive predictive value, TOR termination of resuscitation

a PPV of 0.998 (95 \% CI: 0.995-0.999) for predicting neurologically unfavorable outcomes at 1 month (Table 3). In this same group, the BLS TOR rules had a specificity of 0.859 (95 \% CI: 0.766-0.925), a FPR of $14.1 \%$ (95 \% CI: 7.5-23.4 \%), and a PPV of 0.994 (95 \% CI: 0.990-0.997) for mortality at 1 month (Table 4). The ALS TOR rule for patients with OHCA from noncardiac etiologies had a specificity of 0.833 (95 \% CI: 0.586-0.964), a FPR of 16.7 \% (95 \% CI: 3.6-41.4 \%), and a PPV of 0.996 (95\% CI: 0.988-0.999) for predicting neurologically unfavorable outcomes at 1 month (Table 3). For 1-month mortality, the ALS TOR rule had a specificity of 0.857 (95\% CI: 0.759-0.926), a FPR of $14.3 \%$ (95 \% CI: 7.4-24.1\%), and a PPV of 0.985 (95 \% CI: 0.974-0.993) (Table 4).

\section{Characteristics of unexpected survivors with neurologically favorable outcome}

Seven and six unexpected survivors had good neurological outcomes in the BLS and ALS TOR rules, respectively (see Additional file 1: Table S1). Of these 13 cases, incidental hypothermia, pulmonary embolism, drug overdose, and suffocation accounted for seven cases with noncardiac etiologies. Extracorporeal cardiopulmonary resuscitation (ECPR) was performed in five cases.

\section{Discussion}

We investigated the diagnostic accuracy of the BLS and ALS TOR rules for predicting unfavorable neurological outcomes and mortality 1 month after OHCA using data from SOS-KANTO 2012. Both TOR rules showed a high specificity and PPV and a low FPR in patients with OHCA of cardiac etiology. However, the TOR rules had a high PPV but low specificity and high FPR in patients with OHCA of noncardiac etiology. The imprecision of the BLS and ALS TOR rules is summarized in Table 5.

For patients in the cardiac etiology group, the diagnostic accuracy of the TOR rules was similar to results reported in prior studies conducted in North America and Japan [7, 9-11]. Similarly, the effectiveness of both TOR rules for predicting neurologically unfavorable outcomes and death after OHCA in Japan was demonstrated in this present study. In cases of OHCA due to cardiac etiology, both TOR rules were useful in determining when to terminate resuscitation efforts in the prehospital setting.

This study had several strengths, particularly regarding the population and sample size. Firstly, the efficacy of the TOR rules was validated in all patients transported to emergency hospitals in a densely populated area of 
Table 4 Diagnostic accuracy of termination of resuscitation rules for 1-month mortality

\begin{tabular}{|c|c|c|c|c|c|c|c|}
\hline Etiology of cardiac arrest & Deaths & Survival & Sensitivity (95 \% Cl) & Specificity (95 \% Cl) & FPR $(95 \%$ Cl) & PPV $(95 \%$ Cl) & NPV $(95 \% \mathrm{Cl})$ \\
\hline \multicolumn{8}{|l|}{ BLS TOR rule } \\
\hline \multicolumn{8}{|l|}{ All types } \\
\hline Met criteria & 4165 & 28 & 0.820 & 0.918 & $8.2 \%$ & 0.993 & 0.255 \\
\hline Did not meet criteria & 917 & 314 & $(0.809-0.830)$ & $(0.884-0.945)$ & $(5.5-11.6 \%)$ & (0.990-0.996) & $(0.231-0.280)$ \\
\hline \multicolumn{8}{|l|}{ Cardiac etiology } \\
\hline Met criteria & 2044 & 16 & 0.798 & 0.938 & $6.2 \%$ & 0.992 & 0.318 \\
\hline Did not meet criteria & 517 & 241 & $(0.782-0.814)$ & $(0.901-0.964)$ & (3.6-9.9\%) & $(0.987-0.996)$ & $(0.285-0.352)$ \\
\hline \multicolumn{8}{|l|}{ Noncardiac etiology } \\
\hline Met criteria & 2121 & 12 & 0.841 & 0.859 & $14.1 \%$ & 0.994 & 0.154 \\
\hline Did not meet criteria & 400 & 73 & $(0.826-0.855)$ & $(0.766-0.925)$ & $(7.5-23.4 \%)$ & $(0.990-0.997)$ & $(0.123-0.190)$ \\
\hline \multicolumn{8}{|l|}{ ALS TOR rule } \\
\hline \multicolumn{8}{|l|}{ All types } \\
\hline Met criteria & 1681 & 20 & 0.287 & 0.913 & $8.7 \%$ & 0.988 & 0.048 \\
\hline Did not meet criteria & 4170 & 210 & $(0.276-0.299)$ & $(0.869-0.946)$ & $(5.4-13.1 \%)$ & $(0.982-0.993)$ & $(0.042-0.055)$ \\
\hline \multicolumn{8}{|l|}{ Cardiac etiology } \\
\hline Met criteria & 947 & 9 & 0.299 & 0.941 & $5.9 \%$ & 0.991 & 0.061 \\
\hline Did not meet criteria & 2220 & 144 & $(0.283-0.315)$ & $(0.891-0.973)$ & $(2.7-10.9 \%)$ & $(0.982-0.996)$ & $(0.052-0.071)$ \\
\hline \multicolumn{8}{|l|}{ Noncardiac etiology } \\
\hline Met criteria & 734 & 11 & 0.273 & 0.857 & $14.3 \%$ & 0.985 & 0.033 \\
\hline Did not meet criteria & 1950 & 66 & $(0.257-0.291)$ & $(0.759-0.926)$ & (7.4-24.1\%) & $(0.974-0.993)$ & $(0.025-0.042)$ \\
\hline
\end{tabular}

$A L S$ advanced life support, $B L S$ basic life support, $C I$ confidence interval, $F P R$ false-positive rate, $N P V$ negative predictive value, $P P V$ positive predictive value, TOR termination of resuscitation

Japan. Secondly, we included almost all OHCA patients in this area of Japan because EMS personnel in Japan are legally obligated to transport OHCA patients to the hospital, except in cases of obvious mortality [19, 25]. In previous studies of this topic, TOR occurred in approximately $17 \%$ of patients in the prehospital setting [10].

Table 5 Imprecision of basic and advanced life support termination of resuscitation rules

\begin{tabular}{lll}
\hline \multicolumn{2}{c}{ Etiology } & Imprecision \\
\hline BLS TOR rule & Cardiac & Acceptable \\
ALS TOR rule & Concardiac & Serious \\
& Noncardiac & Serious \\
For predicting 1-month mortality & Serious \\
BLS TOR rule & Cardiac & \\
& Noncardiac & Acceptable \\
ALS TOR rule & Cardiac & Serious \\
& Noncardiac & Serious \\
\hline
\end{tabular}

Imprecision is classified as acceptable and serious when the upper limit of the $95 \%$ confidence interval of the false-positive rate is $\leq 10 \%$ and $>10 \%$, respectively

ALS advanced life support, BLS basic life support, TOR termination of resuscitation
For patients in the noncardiac etiology group, the specificity of both TOR rules for neurologically unfavorable outcomes and mortality at 1 month were relatively low. Conversely, the PPVs of both TOR rules for patients in the noncardiac etiology group were very high and similar to the PPVs for patients in the cardiac etiology group. The pretest probability for 1-month mortality and unfavorable outcomes was $97.0 \%$ and $98.8 \%$, respectively; therefore a high PPV depends on a high pretest probability.

The etiologies of cardiac arrest were categorized clinically according to the Utstein-style guidelines for cardiac arrest data reporting [20]. Although the survival rate for patients with OHCA of noncardiac etiology is very poor, survival rates differ extensively for patients with OHCA of noncardiac etiology [26]. For instance, in patients with OHCA resulting from respiratory disease, asphyxia, drug overdose, and incidental hypothermia, the survival rate is higher than in those from other noncardiac etiologies [26]. In the present study, special circumstances, such as incidental hypothermia or drug overdose, were relatively common etiologies of noncardiac unexpected survivors. The ERC guideline also recommends that EMS personnel should consider continuing CPR during transport to hospital in patients with OHCAs with presumed reversible causes, 
such as drug overdose and hypothermia [12]. Moreover, it is often difficult to determine the etiology of cardiac arrest in the prehospital setting [27]. Costeffectiveness is very important in the TOR rule. Previous researchers have reported that applying the TOR rules reduces approximately $40-50 \%$ of futile transportation and further resuscitation efforts [7, 9]. However, the decision of the TOR is more challenging in contrast to other interventions. It has been argued that success rates of $<1 \%$ still justify the resuscitation effort [12]. According to our results, the TOR rules are less useful for OHCAs of noncardiac etiology than those of cardiac etiology. Until these discrepancies are resolved, it would be necessary to develop new TOR rules that are effective for OHCAs from both cardiac and noncardiac etiologies. At the least, we consider that the TOR rules should not be applied in cases of presumed reversible etiologies.

According to our results, the upper limit of the FPR of the ALS TOR rule was near $10 \%$, which is high for cardiac etiology. As advanced therapies (i.e., ECPR) become widely available, TOR rules require integration with guidance on suitability for these therapies [10, 28, 29]. For example, the low end-tidal carbon dioxide $\left(\mathrm{EtCO}_{2}\right)$ value during CPR may indicate a poor prognosis [30-32]. An $\mathrm{EtCO}_{2}<10 \mathrm{mmHg}$ after 20 minutes of CPR may be considered a component of a multimodal approach to TOR, particular for withholding ECPR [33, 34]. Although $\mathrm{EtCO}_{2}$ was not evaluated in the present study, several unexpected survivors underwent ECPR. Therefore, consideration of $\mathrm{EtCO}_{2}$ may improve the ALS TOR rule.

This study had several limitations. Firstly, this was an observational study, not an interventional study. Although prehospital TOR is not legally permitted in Japan, resuscitation efforts might not be conducted consistently in all OHCA patients. Secondly, SOS-KANTO 2012 was not a population-based study because it was conducted only in the Kanto area of Japan. Therefore, it is possible that our findings are not generalizable to other parts of Japan or other countries. However, the characteristics and outcomes of OHCA patients in this study were similar to those in other populationbased studies in Japan and other countries $[7,10,11,18]$. Finally, this study excluded pediatric patients and those with traumatic cardiac arrest; therefore, the effectiveness of the prehospital TOR rules for these populations is unclear.

\section{Conclusions}

The BLS and ALS TOR rules were useful for predicting neurologically unfavorable outcomes and mortality 1 month after the onset of OHCA of cardiac etiology. However, these rules should be applied cautiously to patients with OHCA of noncardiac etiology.

\section{Key messages}

- The BLS and ALS TOR rules were useful for predicting unfavorable neurological outcomes 1 month after onset of OHCA of cardiac etiology, demonstrating both a high specificity and PPV.

- The TOR rules should be applied with caution to patients with OHCA of noncardiac etiologies because these rules were observed to have a high PPV but a relatively low specificity.

\section{Additional file}

Additional file 1: Table S1. Presenting the characteristics of 13 unexpected survivors with favorable neurological outcomes. (DOCX $16 \mathrm{~kb}$ )

\section{Abbreviations}

AHA: American Heart Association; ALS: Advanced life support; BLS: Basic life support; Cl: Confidence interval; CPR: Cardiopulmonary resuscitation;

ECPR: Extracorporeal cardiopulmonary resuscitation; ELST: Emergency lifesaving technician; EMS: Emergency medical service; ERC: European Resuscitation Council; $\mathrm{EtCO}_{2}$ : End-tidal carbon dioxide; FPR: False-positive rate; OHCA: Out-ofhospital cardiac arrest; PPV: Positive predictive value; ROSC: Return of spontaneous circulation; SOS-KANTO 2012: Survey of Survivors after Cardiac Arrest in the Kanto Area in 2012; TOR: Termination of resuscitation.

\section{Competing interests}

All authors who participated in the current study are members of the Japanese Association for Acute Medicine of Kanto. The authors declare that they have no conflicts of interest.

\section{Authors' contributions}

MK contributed to the study conception and design, data acquisition, analysis, and interpretation, statistical analysis, and drafting of the manuscript. $\mathrm{YH}$ and $\mathrm{AA}$ contributed to the conception and design of the study and data acquisition and interpretation. AS, YT, NY, KN, AY, and NM contributed to data acquisition. All authors critically revised the manuscript for important intellectual content, provided intellectual input to the study and manuscript, and read and approved the final manuscript.

\section{SOS-KANTO 2012 steering council}

Yokohama City University Medical Center, Kanagawa, Japan (President, Naoto Morimura MD); Nihon University School of Medicine, Tokyo, Japan (Director, Atsushi Sakurai MD); National Cerebral and Cardiovascular Center Hospital,

Osaka, Japan (Director, Yoshio Tahara MD); Tokyo Women's Medical University Hospital, Tokyo, Japan (Arino Yaguchi MD); Nihon University Surugadai Hospital, Tokyo, Japan (Ken Nagao MD); Nippon Medical School Hospital, Tokyo, Japan (Tagami Takashi MD); Japanese Red Cross Maebashi Hospital, Gunma, Japan (Dai Miyazaki MD); National Disaster Medical Center, Tokyo, Japan (Tomoko Ogasawara MD); Keio University Hospital, Tokyo, Japan (Kei Hayashida MD, Masaru Suzuki MD); Tokai University School of Medicine, Kanagawa, Japan (Mari Amino MD); Kimitsu Chuo Hospital, Chiba, Japan (Nobuya Kitamura MD); Juntendo University Nerima Hospital, Tokyo, Japan (Tomohisa Nomura MD); Tokyo Metropolitan Children's Medical Centre, Tokyo, Japan (Naoki Shimizu MD); Tokyo Metropolitan Bokutoh Hospital, Tokyo, Japan (Akiko Akashi MD); National Center of Neurology and Psychiatry, Tokyo, Japan (Naohiro Yonemoto DPH).

\section{SOS-KANTO 2012 study group}

Tokai University School of Medicine (Sadaki Inokuchi MD); St. Marianna University School of Medicine, Yokohama Seibu Hospital (Yoshihiro Masui MD); Koto Hospital (Kunihisa Miura MD); Saitama Medical Center Advanced Tertiary Medical Center (Haruhiko Tsutsumi MD); Kawasaki Municipal Hospital Emergency \& Critical Care Center (Kiyotsugu Takuma MD); Yokohama Municipal Citizen's Hospital (Ishihara Atsushi MD); Japanese Red Cross Maebashi Hospital (Minoru Nakano MD); Juntendo University Urayasu Hospital (Hiroshi Tanaka MD); Dokkyo Medical University Koshigaya Hospital (Keiichi Ikegami MD); 
Hachioji Medical Center of Tokyo Medical University (Takao Arai MD); Tokyo Women's Medical University Hospital (Arino Yaguchi MD); Kimitsu Chuo Hospital (Nobuya Kitamura MD); Chiba University Graduate School of Medicine (Shigeto Oda MD); Saiseikai Utsunomiya Hospital (Kenji Kobayashi MD); Mito Saiseikai General Hospital (Takayuki Suda MD); Dokkyo Medical University (Kazuyuki Ono MD); Yokohama City University Medical Center (Naoto Morimura MD); National Hospital Organization Yokohama Medical Center (Ryosuke Furuya MD); National Disaster Medical Center (Yuichi Koido MD); Yamanashi Prefectural Central Hospital (Fumiaki Iwase MD); Surugadai Nihon University Hospital (Ken Nagao MD); Yokohama Rosai Hospital (Shigeru Kanesaka MD); Showa General Hospital (Yasusei Okada MD); Nippon Medical School Tamanagayama Hospital (Kyoko Unemoto MD); Tokyo Women's Medical University Yachiyo Medical Center (Tomohito Sadahiro MD); Awa Regional Medical Center (Masayuki Iyanaga MD); Todachuo General Hospital (Asaki Muraoka MD); Japanese Red Cross Medical Center (Munehiro Hayashi MD); St. Luke's International Hospital (Shinichi Ishimatsu MD); Showa University School of Medicine (Yasufumi Miyake MD); Totsuka Kyoritsu Hospital 1 (Hideo Yokokawa MD); St. Marianna University School of Medicine (Yasuaki Koyama MD); National Hospital Organization Mito Medical Center (Asuka Tsuchiya MD); Tokyo Metropolitan Tama Medical Center (Tetsuya Kashiyama MD); Showa University Fujigaoka Hospital (Munetaka Hayashi MD); Gunma University Graduate School of Medicine (Kiyohiro Oshima MD); Saitama Red Cross Hospital (Kazuya Kiyota MD); Tokyo Metropolitan Bokutoh Hospital (Yuichi Hamabe MD); Nippon Medical School Hospital (Hiroyuki Yokota MD); Keio University Hospital (Shingo Hori MD); Chiba Emergency Medical Center (Shin Inaba MD); Teikyo University School of Medicine (Tetsuya Sakamoto MD); Japanese Red Cross Musashino Hospital (Naoshige Harada MD); National Center for Global Health and Medicine Hospital (Akio Kimura MD); Tokyo Metropolitan Police Hospital (Masayuki Kanai MD); Medical Hospital of Tokyo Medical and Dental University (Yasuhiro Otomo MD); Juntendo University Nerima Hospital (Manabu Sugita MD); Nihon University School of Medicine (Kosaku Kinoshita MD); Toho University Ohashi Medical Center (Takatoshi Sakurai MD); Saiseikai Yokohamashi Tobu Hospital (Mitsuhide Kitano MD); Nippon Medical School Musashikosugi Hospital (Kiyoshi Matsuda MD); Tokyo Rosai Hospital (Kotaro Tanaka MD); Toho University Omori Medical Center (Katsunori Yoshihara MD); Hiratsuka City Hospital (Kikuo Yoh MD); Yokosuka Kyosai Hospital (Junichi Suzuki MD); Saiseikai Yokohamashi Nambu Hospital (Hiroshi Toyoda MD); Nippon Medical School Chiba Hokusoh Hospital (Kunihiro Mashiko MD); Tokyo Metropolitan Children's Medical Centre (Naoki Shimizu MD); National Medical Center for Children and Mothers (Takashi Muguruma MD); Chiba Aoba Municipal Hospital (Tadanaga Shimada MD); Kuki General Hospital (Yoshiro Kobe MD); Matsudo City Hospital (Tomohisa Shoko MD); Japanese Red Cross Narita Hospital (Kazuya Nakanishi MD); Tokyo Bay Urayasu/lchikawa Medical Center (Takashi Shiga MD); NTT Medical Center Tokyo (Takefumi Yamamoto MD); Tokyo Saiseikai Central Hospital (Kazuhiko Sekine MD); Fuji Heavy Industries Health Insurance Society OTA Memorial Hospital (Shinichi Izuka MD); http://www.jaam-kanto.jp/ sos_kanto/sos_kanto2012_contributors.html.

\section{Author details}

'Tertiary Emergency Medical Center, Tokyo Metropolitan Bokutoh Hospital, 4-23-15 Kotobashi, Sumida-ku 130-8575Tokyo, Japan. ${ }^{2}$ Division of Emergency and Critical Care Medicine, Department of Acute Medicine, Nihon University School of Medicine, 30-1 Oyaguchikamicho, Itabashi-ku 173-0032Tokyo, Japan. ${ }^{3}$ National Cerebral and Cardiovascular Center Hospital, 5-7-1 Fujishiro-dai, Suita 565-85650saka, Japan. ${ }^{4}$ National Center of Neurology and Psychiatry, 4-1-1 Ogawa-Higashi, Kodaira 187-8551Tokyo, Japan. ${ }^{5}$ Nihon University Surugadai Hospital, 1-6 Kanda-Surugadai, Chiyoda-ku 101-8309Tokyo, Japan. ${ }^{6}$ Department of Critical Care and Emergency Medicine, Tokyo Women's Medical University Hospital, 8-1 Kawadacho, Shinjuku-ku 162-8666Tokyo, Japan. ${ }^{7}$ Department of Emergency Medicine, Yokohama City University Medical Center, 4-57 Urafunecho, Minami-ku, Yokohama-City 232-0024Kanagawa, Japan.

\section{Received: 29 November 2015 Accepted: 10 February 2016} Published online: 01 March 2016

\section{References}

1. Berdowski J, Berg RA, Tijssen JG, Koster RW. Global incidences of out-ofhospital cardiac arrest and survival rates: systematic review of 67 prospective studies. Resuscitation. 2010;81:1479-87.
2. Mozaffarian D, Benjamin EJ, Go AS, Arnett DK, Blaha MJ, Cushman M, et al. Heart disease and stroke statistics - 2015 update: a report from the American Heart Association. Circulation. 2015;131:e29-322.

3. Sasson C, Rogers MA, Dahl J, Kellermann AL. Predictors of survival from out-of-hospital cardiac arrest: a systematic review and meta-analysis. Circ Cardiovasc Qual Outcomes. 2010;3:63-81.

4. Kitamura T, Iwami T, Kawamura T, Nitta M, Nagao K, Nonogi H, et al. Nationwide improvements in survival from out-of-hospital cardiac arrest in Japan. Circulation. 2012;126:2834-43.

5. Maguire BJ, Hunting KL, Smith GS, Levick NR. Occupational fatalities in emergency medical services: a hidden crisis. Ann Emerg Med. 2002;40:625-32.

6. Mancini ME, Diekema DS, Hoadley TA, Kadlec KD, Leveille MH, McGowan JE, et al. Part 3: ethical issues: 2015 American Heart Association Guidelines update for cardiopulmonary resuscitation and emergency cardiovascular care. Circulation. 2015;132:S383-96.

7. Morrison LJ, Visentin LM, Kiss A, Theriault R, Eby D, Vermeulen M, et al. Validation of a rule for termination of resuscitation in out-of-hospital cardiac arrest. N Engl J Med. 2006;355:478-87.

8. Morrison LJ, Verbeek PR, Vermeulen MJ, Kiss A, Allan KS, Nesbitt L, et al. Derivation and evaluation of a termination of resuscitation clinical prediction rule for advanced life support providers. Resuscitation. 2007;74:266-75.

9. Morrison LJ, Verbeek PR, Zhan C, Kiss A, Allan KS. Validation of a universal prehospital termination of resuscitation clinical prediction rule for advanced and basic life support providers. Resuscitation. 2009;80:324-8.

10. Sasson C, Hegg AJ, Macy M, Park A, Kellermann A, McNally B, et al. Prehospital termination of resuscitation in cases of refractory out-of-hospital cardiac arrest. JAMA. 2008;300:1432-8.

11. Kajino K, Kitamura T, Iwami T, Daya M, Ong MEH, Hiraide A, et al. Current termination of resuscitation (TOR) guidelines predict neurologically favorable outcome in Japan. Resuscitation. 2013;84:54-9.

12. Bossaert LL, Perkins GD, Askitopoulou H, Raffay VI, Greif R, Haywood KL, et al. European Resuscitation Council Guidelines for Resuscitation 2015: Section 11. The ethics of resuscitation and end-of-life decisions. Resuscitation. 2015;95:302-11.

13. Drennan IR, Lin S, Sidalak DE, Morrison LJ. Survival rates in out-of-hospital cardiac arrest patients transported without prehospital return of spontaneous circulation: an observational cohort study. Resuscitation. 2014;85:1488-93.

14. Ruygrok ML, Byyny RL, Haukoos JS, Colorado Cardiac Arrest \& Resuscitation Collaborative Study Group, Denver Metro EMS Medical Directors. Validation of 3 termination of resuscitation criteria for good neurologic survival after out-of-hospital cardiac arrest. Ann Emerg Med. 2009;54:239-47.

15. Fukuda T, Ohashi N, Matsubara T, Doi K, Gunshin M, Ishii T, et al. Applicability of the prehospital termination of resuscitation rule in an area dense with hospitals in Tokyo: a single-center, retrospective, observational study: is the pre hospital TOR rule applicable in Tokyo? Am J Emerg Med. 2014;32:144-9.

16. SOS-KANTO 2012 Study Group. Changes in pre-and in-hospital management and outcomes for out-of-hospital cardiac arrest between 2002 and 2012 in Kanto, Japan: the SOS-KANTO 2012 Study. Acute Med Surg. 2015;2:225-33.

17. Kitamura N, Nakada TA, Shinozaki K, Tahara Y, Sakurai A, Yonemoto N, et al. Subsequent shock deliveries are associated with increased favorable neurological outcomes in cardiac arrest patients who had initially nonshockable rhythms. Crit Care. 2015;19:322.

18. Kitamura T, Iwami T, Kawamura T, Nagao K, Tanaka H, Hiraide A. Nationwide public-access defibrillation in Japan. N Engl J Med. 2010;362:994-1004.

19. Goto Y, Maeda T, Nakatsu-Goto Y. Decision tree model for predicting longterm outcomes in children with out-of-hospital cardiac arrest: a nationwide, population-based observational study. Crit Care. 2014;18:R133.

20. Perkins GD, Jacobs IG, Nadkarni VM, Berg RA, Bhanji F, Biarent D, et al. Cardiac Arrest and Cardiopulmonary Resuscitation Outcome Reports: Update of the Utstein Resuscitation Registry Templates for Out-of-Hospital Cardiac Arrest: A Statement for Healthcare Professionals From a Task Force of the International Liaison Committee on Resuscitation (American Heart Association, European Resuscitation Council, Australian and New Zealand Council on Resuscitation, Heart and Stroke Foundation of Canada, InterAmerican Heart Foundation, Resuscitation Council of Southern Africa, Resuscitation Council of Asia); and the American Heart Association Emergency Cardiovascular Care Committee and the Council on Cardiopulmonary, Critical Care, Perioperative and Resuscitation. Circulation. 2015;132:1286-300.

21. Jennett B, Bond M. Assessment of outcome after severe brain damage. Lancet. 1975;1:480-4.

22. Cummins RO, Chamberlain DA, Abramson NS, Allen M, Baskett PJ, Becker L, et al. Recommended guidelines for uniform reporting of data from out-of- 
hospital cardiac arrest: the Utstein Style. A statement for health professionals from a task force of the American Heart Association, the European Resuscitation Council, the Heart and Stroke Foundation of Canada, and the Australian Resuscitation Council. Circulation. 1991:84:960-75.

23. Callaway CW, Donnino MW, Fink EL, Geocadin RG, Golan E, Kern KB, et al. Part 8: Post-Cardiac Arrest Care: 2015 American Heart Association Guidelines Update for Cardiopulmonary Resuscitation and Emergency Cardiovascular Care. Circulation. 2015;132:5465-82

24. Sandroni C, Cariou A, Cavallaro F, Cronberg T, Friberg H, Hoedemaekers C, et al. Prognostication in comatose survivors of cardiac arrest: An advisory statement from the European Resuscitation Council and the European Society of Intensive Care Medicine. Resuscitation. 2014;85:1779-89.

25. Goto Y, Maeda T, Goto YN. Termination-of-resuscitation rule for emergency department physicians treating out-of-hospital cardiac arrest patients: an observational cohort study. Crit Care. 2013;17:R235.

26. Kitamura T, Kiyohara K, Sakai T, Iwami T, Nishiyama C, Kajino K, et al. Epidemiology and outcome of adult out-of-hospital cardiac arrest of noncardiac origin in Osaka: a population-based study. BMJ Open. 2014;4:e006462

27. Kürkciyan I, Meron G, Behringer W, Sterz F, Berzlanovich A, Domanovits $H_{\text {, }}$ et al. Accuracy and impact of presumed cause in patients with cardiac arrest. Circulation. 1998;98:766-71.

28. Zive D, Koprowicz K, Schmidt T, Stiell I, Sears G, Van Ottingham L, et al. Variation in out-of-hospital cardiac arrest resuscitation and transport practices in the Resuscitation Outcomes Consortium: ROC Epistry-Cardiac Arrest. Resuscitation. 2011:82:277-84.

29. Stub D, Bernard S, Pellegrino V, Smith K, Walker T, Sheldrake J, et al. Refractory cardiac arrest treated with mechanical CPR, hypothermia, ECMO and early reperfusion (the CHEER trial). Resuscitation. 2015;86:88-94.

30. Levine RL, Wayne MA, Miller CC. End-tidal carbon dioxide and outcome of out-of-hospital cardiac arrest. N Engl J Med. 1997;337:301-6.

31. Cantineau JP, Lambert Y, Merckx P, Reynaud P, Porte F, Bertrand C, et al. End-tidal carbon dioxide during cardiopulmonary resuscitation in humans presenting mostly with asystole: a predictor of outcome. Crit Care Med. 1996;24:791-6

32. Ahrens T, Schallom L, Bettorf K, Ellner S, Hurt G, O'Mara V, et al. End-tidal carbon dioxide measurements as a prognostic indicator of outcome in cardiac arrest. Am J Crit Care. 2001;10:391-8.

33. Soar J, Nolan JP, Böttiger BW, Perkins GD, Lott C, Carli P, et al. European Resuscitation Council Guidelines for Resuscitation 2015: Section 3. Adult advanced life support. Resuscitation. 2015;95:100-47.

34. Link MS, Berkow LC, Kudenchuk PJ, Halperin HR, Hess EP, Moitra VK, et al. Part 7: Adult Advanced Cardiovascular Life Support: 2015 American Heart Association Guidelines Update for Cardiopulmonary Resuscitation and Emergency Cardiovascular Care. Circulation. 2015;132:S444-64.

\section{Submit your next manuscript to BioMed Central and we will help you at every step:}

- We accept pre-submission inquiries

- Our selector tool helps you to find the most relevant journal

- We provide round the clock customer support

- Convenient online submission

- Thorough peer review

- Inclusion in PubMed and all major indexing services

- Maximum visibility for your research

Submit your manuscript at www.biomedcentral.com/submit

CBiomed Central 